\title{
An Adolescent Boy with Acquired Epileptic Aphasia -Landau Kleffner Syndrome: A rare case report
}

\author{
Sharma $\mathrm{SR}^{1}$, Sharma $\mathrm{N}^{2}$, Yeolekar $\mathrm{ME}^{3}$ \\ ${ }^{1}$ Dr. Shri Ram Sharma, MBBS, MD. DM. (Neurology), Assistant Professor in Neurology, ${ }^{2}$ Dr. Nalini Sharma, ${ }^{3}$ Dr. \\ ME Yeolekar Professor in Neurology. All from North Eastern Indira Gandhi Regional Institute of Medical Sciences \\ (NEIGRIHMS) Shillong, India.
}

Address for correspondence: Dr. SR Sharma, E-mail: srmsims_sharma@rediffmail

\begin{abstract}
A 13 year adolescent boy presented with complaints of progressive loss of speech and seizure disorder for the past 1 year. The boy was developmently normal before the onset of illness. There was history of subtle trauma to head. He started communicating with signs followed by paucity of speech progressed to complete aphasia and abnormal behavior in the form of burst of aggressiveness and hyperactivity. Boy had partial, generalized tonic-clonic seizures occurred during sleep. Mental status examination revealed abnormal behaviour, attention deficits, auditory agnosia and aphasia (both expressive and receptive). All relevant investigations including BERA were normal.EEG showed repetitive spikes and wave activity in bilateral parietooccipital regions during nonrapid eye movement. Clinical features and EEG led to a diagnosis of Landau Kleffner Syndrome. He was started on Lamotrigine, steroid and speech therapy. The boy started showing improvement of symptoms in form of understanding of short sentences.
\end{abstract}

Key words: Landau Kleffner Syndrome, EEG, Seizures

\section{Introduction}

In 1957, Landau and Kleffner initially described Acquired Epileptic Aphasia (AEA) and subsequently reluctantly agreed to the attachment of their names to the syndrome ${ }^{1}$.This typically develops in healthy children who acutely or progressively lose receptive and expressive language ability coincident with the appearance of paroxysmal Electroencephalographic (EEG) changes ${ }^{2}$. It is a rare childhood epileptic aphasia (AEA) of unknown etiology2. Psychomotor and behavioral problems are also observed ${ }^{3}$. It is imperative that health professionals become alert to the characteristic symptoms of Landau Kleffner syndrome ${ }^{4}$. All children with LKS have abnormal electrical brain waves that can be documented by an EEG ${ }^{5}$. The aim of presentation of the present case is to create awareness about this syndrome.

\section{Case history}

A 13-year adolescent boy of class eight presented to us with the complaint of progressive loss of speech and seizure disorder for the past one year. His parents revealed that the boy was developmentally normal before the onset of illness. He had history of head injury two months before the onset of above symptoms. The immediate two months after head trauma he was uneventful, after which, his speech progressively diminished and he started communicating with signs (non verbal gestures). Over a period of 7 to 8 months, the paucity of speech progressed to complete aphasia and the boy had abnormal behavior in the form of bursts of aggressiveness and hyperactivity. The boy also had five episodes of generalized tonic clonic seizures together with an episode of partial seizure that occurred during sleep. Boy also showed abnormal behavior along with attention deficit and did not respond verbally to any command.

The general and systemic examinations were normal except for speech and language. Objective testing of all aspects of speech such as comprehension, repetition, naming, fluency, reading, and writing was done by a qualified speech pathologist. Both bedside 
and office testing of language skills was supplemented by formal neuropsychological testing that revealed auditory agnosia and aphasia (both receptive and expressive). The first manifestation of language problem was difficulty understanding what was said (word deafness or auditory verbal agnosia) and lack of recognition of familiar noises, alert responses to sound was normal. Receptive language was severely impaired. Speech production was affected equally as language comprehension. Reading and writing was remarkably preserved initially. Speech disturbance included use of jargon or nonsense, paraphasia, asyntaxia and verbal sterotypies. Later on the boy had difficulty telling the differences between other non verbal based sounds such as the door bell ringing or a dog barking. Speech became garbled and he started communicating mainly through signs. Language deterioration occurred over months. Then abnormal behavior, attention deficit was also noticed. Hematology, Liver funtion tests, renal function tests, Cerebrospinal fluid examination, BERA, and Fundus was normal. The EEG (Fig. 1) revealed repetitive spike and wave activity which was seen bilaterally in the parieto occipital regions. Sleep activated the findings of EEG, MRI was also normal. On the basis of presentation, EEG findings and normal MRI; Landau Kleffner syndrome was diagnosed and the boy was started on Lamotrigine and steroid (prednisolone) along with speech therapy. The boy started showing gradual improvement in symptoms in the form of understanding short sentences.

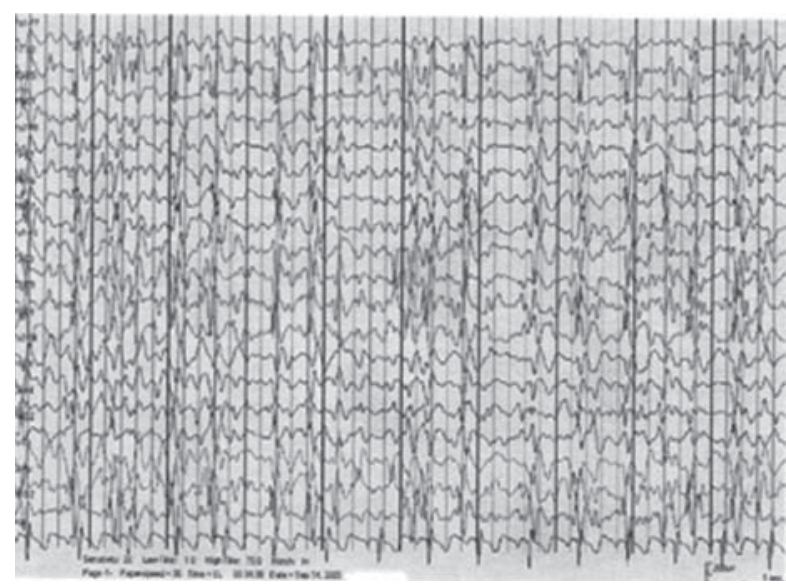

Fig 1: Showing continuous spike wave discharges during sleep(CSWDS) in the patient.

\section{Discussion}

Landau Kleffner syndrome also known as Worster Drought syndrome is characterized by a triad of receptive and expressive aphasia, EEG abnormalities and in a majority of cases, epileptic seizures in previously normal child, usually a boy ${ }^{1}$. Landau Kleffner syndrome may also be called acquired epileptic aphasia or infantile acquired aphasia, aphasia with convulsive disorder ${ }^{4,5}$. This syndrome was first described by Dr. William M.Landau and Dr. Frank R. Kleffner, who identified six children with this disorder ${ }^{1}$. More than 200 cases have been described in global literature ${ }^{7,8}$. A slight predominance in boys is noted, with a male to female ratio of $1.7: 1^{9}$. Aphasia usually appears at 4-7 years of age. However symptom onset has been described in patients as young as 18 months and in those as old as 13 years ${ }^{5,6}$. Landau Kleffner syndrome occurs most frequently in normally developing children, these children have trouble understanding what is said to them. Doctors often refer to this problem as auditory agnosia or" word deafness". The auditory agnosia may occur slowly or very quickly ${ }^{7,8}$. Parents often think that the child is developing a hearing problem or has become suddenly deaf. Hearing tests however show normal hearing ${ }^{10}$.

The prevalence of clinical seizures in LKS is $70-85 \%$. Among patients in whom ictal semiology is well described, $59 \%$ had partial seizures, $39 \%$ had generalized tonic-clonic seizures, and $16 \%$ had atypical absences. Myoclonic seizures involving the face and eyes have also been described ${ }^{7,8}$. Seizures may be frequent or repetitive (nocturnal) with rare episodes of convulsive and nonconvulsive status epilepticus. About $12 \%$ of patients have a family history of epilepsy ${ }^{3,10}$.

Behavioral disturbances are seen in as many as $78 \%$ of the patients ${ }^{8}$. The diagnosis of autism is often considered because of the common presence of asyntaxia parapsias and verbal stereotypies. Hyperactivity and decreased attention span are observed in as many as $80 \%$ of patients ${ }^{7}$. Aggressive and oppositional behavior including raged attacks, personality disturbances and intellectual deficits are common and may be wrongly looked as manifestations of psychosis ${ }^{11}$. Explanation for this may include a primary functional disinhibition at limbic or diencephlic level or as a secondary (frustration induced) effect due to loss of comprehension ${ }^{6}$.

The accurate Neurophysiologic mechanism of stagnation involution of acquired speech in patients with LKS is not completely clear yet. Some authors believe a functional disconnection of brain cortex areas responsible by reception language (temporal medial cortex) and cortex areas responsible by spoken language, in the junction of frontal temporal and parietal in the left brain hemisphere ${ }^{12}$. How much speech commitment is resulting from morphologic changes in cortex microstructure and how much is related exclusively to functional changes is not totally cleared ${ }^{13}$. In one study, 13 of the 19 patients had perisylvian magnet-encephalography $(\mathrm{MEG})^{14}$. The epileptic form 
activity in LKS is thought to result in functional ablation of eloquent speech areas ${ }^{4}$.

A few cases of secondary LKS have been described in low grade brain tumors, close head injury, neurocysticercosis, CNS vasculitis, mitochondrial respiratory chain complex I deficiency etc ${ }^{15,16}$.

EEG abnormalities are constantly found in those without clinical seizures ${ }^{12}$ and they are believed to be cortical manifestations of a lower sub cortical differentiating process ${ }^{15}$. EEG abnormalities consist of discharge of spikes, spike-wave or sharp waves of high amplitude either synchronous in both hemispheres, or in foci of variable localization, generally temporal, temporooccipital. They are not influenced by hyperventilation or photicstimuli12

Sleep usually activates the abnormalities and in some cases the abnormalities are seen only in sleep recordings ${ }^{7,8}$. Thus a child suspected of LKS should have an EEG during sleep (REM) particularly if awake record is normal. If the sleep EEG is normal a high index of suspicion for the diagnosis of LKS continues, the child should be monitored with prolonged EEG Recording ${ }^{17}$ and specific neuroimaging studies such as CT and MRI studies, which typically yields normal results, and PET scan has demonstrated either uni or bilateral hypo or hyper metabolism ${ }^{18}$. Microscopic examination of surgical specimen has shown minimal gliosis but no evidence of encephalitis.

The diagnosis of LKS largely depends on being aware that the condition exists, its pattern of presentation differential diagnosis includes autism, pervasive developmental disorder, hearing impairment, learning disability, auditory and or verbal processing disorder, attention deficit disorder, mental retardation, childhood schizophrenia, or emotional/behavioral problems ${ }^{19}$.

While many of the affected individuals have clinical seizures, some only have electrographic status epilepticus of sleep (ESES) ${ }^{19}$. Continuous spike wave discharges during sleep (CSWDS) mimics LKS. Many current researchers classify LKS as part of the syndrome of electrical status epilepticus during sleep, which is also known as continuous spike and wave of slow-wave sleep ${ }^{17}$. The distinguishing feature of CSWDS is the continuous bilateral and diffuse slow spike-wave activity persisting through all of the slow sleep stages. The spike-wave index ranges from $85 \%$ to $100 \%{ }^{17,20}$.

Treatment of LKS can be frustrating. Standard antiepileptic drugs such as valproate and lamotrigine ${ }^{22}$ can sometimes reduce seizure frequency and improve language and cognitive function. If the seizure and aphasia persist, a trial of steroids should be considerd. One recommended schedule consist of oral prednisolone, $2 \mathrm{mg} / \mathrm{kg} /$ day for 2 months than prednisolone is slowly tapered until the child is taking $0.5 \mathrm{mg} / \mathrm{kg}$ every other day. If corticosteroids are beneficial, this dosage is usually maintained for 6-8 months ${ }^{21}$. In addition Intravenous immunoglobulin is drug of choice ${ }^{20}$. It is imperative to evaluate speech therapy and maintain treatment for several years, because improvement in language function occurs only over a prolonged period ${ }^{23}$.Subpial cortical transaction have been reported to useful in clinically refractory cases with $\mathrm{LKS}^{13}$.

Conclusion: Although LKS is uncommon, there is a need for an increased awareness of the disorder, particularlary among neurologist to whom children are referred with a rapid loss of speech and language in a previously normal child. Once considered; the diagnosis can be confirmed by sleep EEG activity. A short course of steroid would seem reasonable, along with antiepileptic drugs. Speech therapy and educational rehabilitation should be started as early as possible and a neurosurgical referral should be considered for those children with persisting aphasia and drug resistant seizures.

\section{References}

1. Landau WM, Kleffner FR-Syndrome of acquired aphasia with convulsive disorder.Neurology 1957;7:523-30.

2. Commission on classification and terminology of the International League Against Epilepsy. Proposal for revised classification of epilepsies and epileptic syndromes. Epilepsia 1989;30:389-99.

3. Roulet Perez E, Davidoff U, Despland P, Deonna T. Mental and behavioral Deterioration of children with epilepsy and CSWS; Acquired epileptic frontal syndrome. Dev Med Child Neurol 1993;35:661-74.

4. Raybarman C. Landau Kleffner syndrome: a case report. Neurolindia 2002:50: 212-3 Bishop DV.Age of onset and outcome in acquired aphasia with convulsive disorder

5. (Landau Kleffner syndrome). Dev Med Child Neurol 1985;705-12.

6. Bharadwaj p Sharma R, Gautam P. Acquired epileptic aphasia: Landau-Kleffner Syndrome. J Pediatr Neurosci 2009;4:52-3. 
7. Tharpe AM, Olson BJ. Landau-Kleffner syndrome: acquired epileptic aphasia in children. J Am Acad Audiol 1994;5:146-50.

8. Tuchman RF. Acquired epileptiform aphasia. Semin Pediatr Neurol 1997;4:93-101.

9. Robinson RO, Baird G, Robinson G, Simonoff E.Landau KleffnerSyndrome: course and correlates with outcome. Dev Med Child Neurol 2001;43:2437.

10. Lanzi G, Vegetate P, Conte S, et al. A correlated fluctuation of language and EEG abnormalities in a case of the Landau-Kleffner syndrome. Brain Dev 1994;16:329-34.

11. Bhatia M.S,Khanna S, Chaudhary S. Landau Kleffner Syndrome. Indian $J$ Pediatrics 1995;35:911.

12. Paulo Breno Noranho Liberalesso, Lincoln Francisco Nascimento, Karlin Fabianne, Ari Leon Jurkiewicz,Bianca Simone Zeigelboim. LandauKleffner syndrome without seizures-would speech delay justify the treatment with antiepileptic drugs? J Epilepsy Clin Neurophysiol 2008;14:1676-82.

13. Morrell F, Whisler WW, Smith MC, et al. LandauKleffner syndrome. Treatment with subpial intracortical transection. Brain 1995;118:1529-46.

14. Paetau R. Magnetoencephalography in LandauKleffner Syndrome. Epilepsia 2009;50:51-4.

15. Appleton RE. The Landau-Kleffner Syndrome. Arch Dis Child 1995;72:386-7.
16. Soprano AM, Garcia EF, Caraballo R, Fejerman N. Acquired epileptic aphasia: neuropsychologic follow-up of 12 patients. Pediatr Neurol 1994;11:230-5.

17. Tassinari CA, Rubboli G, volpi L, Encephalopathy with electrical status epilepticus during slow sleep or ESES syndrome including the acquired aphasia. Clin Neurophysiol 2000;111:S 94-S102.

18. Maquet $P$,Hirsch E,Dive D, Salmon E,Mares Caux C,Frank G. Cerebral glucose Utilization during sleep in Landau Kleffner syndrome. A positron emission tomography (PET) Study. Epilepsia 1990;31:778-83.

19. Rotenberg J, Pearl PL. Landau Kleffner syndrome. Arch Neurol 2003;60:1019-21.

20. ArtsWF, Aarsen FK, Scheltens-deBoerM, CatsmanBerrevoets CE. Landau- Kleffner syndrome and CSWS syndrome: treatment with intravenous immunoglobulin. Epilepsia 2009;50:55-8.

21. Beumonoir A. The Landau Kleffner syndrome in: Roger J, Beureau M, Drovet Ch, Dreifuss FE, Perret A, Wolf P., editors. Epileptic syndrome in infancy, Childhood and adolescence $2^{\text {nd }}$ ed. London, England: John Libbey Eurotext; 1992.231- 43.

22. Haessler F., Gierow B., Buhmann J. Lamotrigine and Landau-Kleffner syndrome: Two cases. Padiatrie und Grenzgebiete 2002; 41:161-67.

23. Duran MH, Guimaraes CA, Medeiros LL, Guerreiro MM. Landau-Kleffner Syndrome: long-term followup. Brain Dev 2009;31:58-63.

\section{How to cite this article?}

Sharma SR, Sharma N, Yeolekar ME. An Adolescent Boy with Acquired Epileptic Aphasia -Landau Kleffner Syndrome. A rare case report. J Nepal Paedtr Soc 2011;31(1):57-60. 